\title{
The Kuopio idiopathic normal pressure hydrocephalus protocol: initial outcome of 175 patients
}

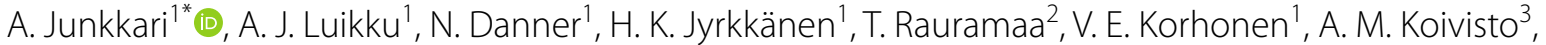 \\ O. Nerg ${ }^{3}$, M. Kojoukhova' ${ }^{1}$,T. J. Huttunen ${ }^{1}$, J. E. Jääskeläinen ${ }^{1}$ and V. Leinonen ${ }^{1,4,5}$
}

\begin{abstract}
Background: The Kuopio University Hospital (KUH) idiopathic normal pressure hydrocephalus (iNPH) cerebrospinal fluid (CSF) shunting protocol is described together with the initial outcomes of 175 patients with probable iNPH treated according to this protocol from a defined population. Our secondary aim was to display the variety of differential diagnoses referred to the KUH iNPH outpatient clinic from 2010 until 2017.

Methods: Patients were divided into four groups according to the prognostic tests: tap test (positive or negative) and infusion test (positive or negative). The short-term outcome was compared between groups. The 3-month outcome following shunt surgery was assessed by measuring gait speed improvement, using a 12-point iNPH grading scale (iNPHGS) and the 15D instrument.

Results: From 341 patients suspected of iNPH, 88 patients were excluded from further research mostly due to deviation from the protocol's gait assessment guidelines. Hence 253 patients with suspected iNPH were included in the study, 177/253 (70\%) of whom were treated with a CSF shunt. A favorable clinical outcome following surgery was observed in $79-93 \%$ of patients depending on the prognostic group. A moderate association (Cramer's $V=0.32$ ) was found between the gait speed improvement rate and the prognostic group $\left(X^{2}, p=0.003\right)$. Patients with a positive tap test had the highest gait speed improvement rate (75\%). In addition, an improvement in walking speed was observed in 4/11 patients who had both a negative tap test and a negative infusion test. Other outcome measures did not differ between the prognostic groups. Conditions other than iNPH were found in $25 \%$ of the patients referred to iNPH outpatient clinic, with the most prevalent being Alzheimer's disease.
\end{abstract}

Conclusions: Our results emphasize the importance of a systematic diagnostic and prognostic workup especially in cases with an atypical presentation of iNPH. Additional diagnostic testing may be required, but should not delay adequate care. Active surgical treatment is recommended in patients with a high clinical probability of iNPH. Other neurological conditions contributed to most of the non iNPH diagnoses.

Keywords: Normal pressure hydrocephalus, Outcome, Tap test, Infusion test, Comorbidity

\footnotetext{
*Correspondence: antti.junkkari@kuh.fi

${ }^{1}$ Neurosurgery of NeuroCenter, Kuopio University Hospital (KUH)

and University of Eastern Finland (UEF), POB 100, 70029 Kuopio, Finland

Full list of author information is available at the end of the article
} 


\section{Introduction}

Idiopathic normal pressure hydrocephalus (iNPH) is a progressive neurological disorder, affecting the aged population, which can be ameliorated by cerebrospinal fluid (CSF) shunting [1, 2]. A suspicion of iNPH rises, when patients exhibit a progressive worsening of gait, cognitive impairment and urinary incontinence, accompanied with ventricular enlargement (ventriculomegaly) demonstrated by computed tomography (CT) or magnetic resonance imaging (MRI) of the brain $[1,2]$. It has been recently hypothesized that ventriculomegaly might be a sign of early neurodegeneration [3]. Since several conditions may feature a similar gait disorder [3, 4] and ventriculomegaly $[3,5]$ thorough diagnostic evaluations should be performed in collaboration with neurologists, neuroradiologists and neurosurgeons [3-6]. Due to the progressive nature of iNPH, patients should be treated without unnecessary delays after establishing the diagnosis, since the condition worsens over time [7, 8]. However, also the response rate for CSF shunting seems to begin to decrease on average after 6 months following surgery [9], possibly indicating the progression of iNPH or its comorbidities [10]. It has also been suggested that a non-sustained response may indicate another condition than iNPH [3].

In three decades the methods and criteria for diagnosing iNPH and predicting the outcome of CSF shunting, while not perfect, have become more robust and less invasive $[1,2]$. During this time, a gradual adaptation to the iNPH guidelines and literature has modified the practice in Kuopio University Hospital's (KUH) NPH outpatient clinic: From 1991 until 2010 the KUH protocol included a 24-h intraventricular pressure monitoring from all patients with suspected iNPH. In early 2010, after the adaptation of tap-test, infusion testing and motivation to decrease risks involved with direct intracranial pressure (ICP) monitoring, a three-step prognostic test protocol was launched (Fig. 1, Table 1), the KUH iNPH protocol. Our aim is to describe KUH iNPH protocol and the initial outcomes of 175 patients with probable iNPH who were treated based on this protocol.

Our secondary objective was to examine whether the patients selected to the treatment on each step of the prognostic test protocol, differentiated from each other in terms of clinical appearance and severity of the illness, and to describe the initial outcomes in each prognostic group. Our tertiary aim was to display the variety of differential diagnoses referred to the KUH iNPH outpatient clinic from 2010 until 2017.

\section{Current prognostic and differential diagnostic tests for iNHP}

The lumbar tap test (LTT) has been used to temporarily emulate the function of a CSF shunt and to predict the outcome of treatment [2,11-13] (Table 1). It has been demonstrated that a larger volume of drained CSF during LTT does not provide additional value [14] but some researchers have suggested that a longer observation time (up to 24-h) may be used to increase the sensitivity of the LTT [15]. In addition to measuring walking speed in the LTT, a timed up and go (TUG) test can also be used $[16,17]$. As an alternative to a single lumbar puncture, another option is to continuously drain CSF over several days removing a total volume of 300$500 \mathrm{ml}$ of CSF, also known as external lumbar drainage (ELD) $[2,13]$. Despite the various test methods, reports on the minimal clinically significant improvement in gait speed after LTT or ELD are scarce [13]. However, patients with a strong clinical suspicion of iNPH but negative results in LTT should not be denied CSF shunt surgery, but undergo further testing of CSF hydrodynamics $[11-13,18]$. For example in iNPH, elevated CSF outflow resistance may be observed in a lumbar infusion test $[13,18-20]$. Even though the diagnostic and prognostic workup of iNPH can be enhanced with the infusion test, there still remain patients with iNPH who potentially could benefit from CSF shunting despite a normal CSF outflow resistance in the infusion test [12, $13,18,20]$. As a further test, continuous direct monitoring of ICP has been used, but in addition to the invasive nature of the procedure, the additional prognostic value of the different abnormalities observed during monitoring, such as B waves or pulsatile ICP, have not been confirmed [2, 13, 21]. Also more sophisticated computerized methods merging multimodal data, such as Disease State Index (DSI), have problems in predicting outcome of CSF shunting in patients with iNPH [22].

\section{Methods \\ Patients}

The permission for the research was received from the Research Ethics Board of KUH. The study was conducted according to the Declaration of Helsinki and all patients provided informed consent. People suspected to suffer from iNPH were primarily examined by a neurologist and referred for further neurosurgical investigations if the patient exhibited one to three symptoms possibly related to NPH (impaired gait, cognition or urinary continence) together with enlarged brain ventricles (Evans' index $>0.3$ ) in CT or MRI (Fig. 1) and without other explicit cause of the symptoms. 
NPH registry and outpatient clinic records 2010-2017

Patients suspected of iNPH

62 Unstandardized tap tests ${ }^{a}$

$15 \mathrm{sNPH}$

5 tap tests failed

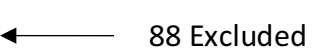

6 infusion tests failed

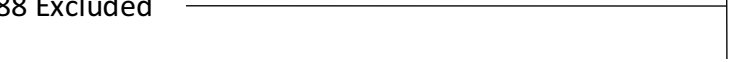

\section{The Kuopio iNPH protocol}

83/98 (85\%) Favorable Clinical outcome

$55 / 73$ (75\%) Walking speed improvement ${ }^{d}$

41/86 (48\%) Favorable iNPHGS outcome ${ }^{e}$

31/59 (52\%) Favorable HRQoL outcome ${ }^{f}$

4 probable iNPH

1 unlikely iNPH ${ }^{\mathrm{g}}$

$98 / 103$

Yes

Shunt

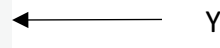

26/28 (93\%) Favorable Clinical outcome

$14 / 24$ (58\%) Walking speed improvement ${ }^{d}$

$10 / 23$ (43\%) Favorable iNPHGS outcome $e^{e}$

7/13 (54\%) Favorable HRQoL outcome

7 possible iNPH

39 unlikely iNPH ${ }^{\text {h }}$

28/34 (82\%) Favorable Clinical outcome ${ }^{c}$

$12 / 29$ (41\%) Walking speed improvement ${ }^{d}$

$11 / 31(36 \%)$ Favorable iNPHGS outcome ${ }^{e}$

$14 / 21(67 \%)$ Favorable HRQoL outcome ${ }^{f}$

3 possible iNPH

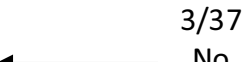

29/75

4
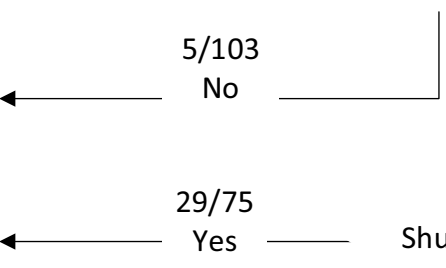

Yes Shunt

$46 / 75$

No

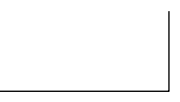

$34 / 37$

$\longleftarrow$ Yes

Yes Shunt

$37 / 75$

$3 / 37$

No

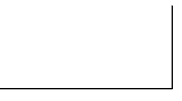

11/14 (79\%) Favorable Clinical outcome ${ }^{c}$ $4 / 11(36 \%)$ Walking speed improvement ${ }^{d}$ $7 / 10(70 \%)$ Favorable iNPHGS outcome ${ }^{e}$ $3 / 7$ (43\%) Favorable HRQoL outcome ${ }^{f}$

22 unlikely iNPH

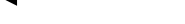

$14 / 36$

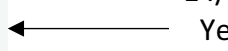

Yes

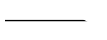

$22 / 36$

2/2 (100\%) Favorable Clinical outcome ${ }^{c}$

$0 / 2(0 \%)$ Walking speed improvement ${ }^{d}$

$1 / 2(50 \%)$ Favorable iNPHGS outcome ${ }^{e}$ $1 / 1(100 \%)$ Favorable HRQoL outcome ${ }^{f}$
No

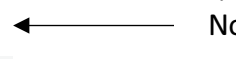

$2 / 2$

Yes $\quad$ Shunt
$103 / 253$

- Positive

Tap test ${ }^{\mathrm{b}}$

1<smiles>C=CC</smiles>

$150 / 253$

Negative

Fig. 1 Flowchart of the study population. The initial outcome 3 months postoperatively has been highlighted in grey in each prognostic group. The number of observations, ratios and percentages have been given to account for any missing data. ${ }^{a}$ Tap test evaluation based only on clinician's judgement. ${ }^{b}$ Description of the performance and interpretation of tap test are found in (Table 1). Improvement of any core symptoms (gait, cognition and urinary incontinence) assessed by neurosurgeon. ${ }^{\mathrm{d} A t}$ least $20 \%$ improvement in average gait speed, gait test task and evaluation described in (Table 1). ${ }^{e}$ Reduction in the iNPHGS total score at least by a single point. ${ }^{f}$ At least 0.015 improvement in $15 \mathrm{D}$ score. ${ }^{9} 1$ Ventriculomegaly (congenital or unclear etiology). h 8 VAD or CVD,7 AD, 7 AD +VAD, 3 Cognitive impairment or dementia of unspecified etiology, 3 Ventriculomegaly (congenital or unclear etiology), 3 PD (2 vascular, 1 idiopathic), 2 Drug induced parkinsonism or psychiatric condition, 2 traumatic brain injury, 1 FTD, 1 Spinal stenosis, 1 arthrosis, 1 LBD. 17 AD, 5 spinal stenosis, 3 Cognitive impairment or dementia of unspecified etiology, 3 VAD or CVD, 2 Drug induced parkinsonism or psychiatric condition, 1 LBD, 1 Vertigo. 'Patients underwent 24-h intraventricular measurement of ICP, interpretation of the monitoring is described in (Table 1). iNPH Idiopathic normal pressure hydrocephalus, sNPH secondary normal pressure hydrocephalus, ICP intracranial pressure, VAD vascular dementia, CVD cerebrovascular disease, AD Alzheimer's disease, PD Parkinson's disease, iPD idiopathic PD, $L B D$ Lewy's bodies disease 


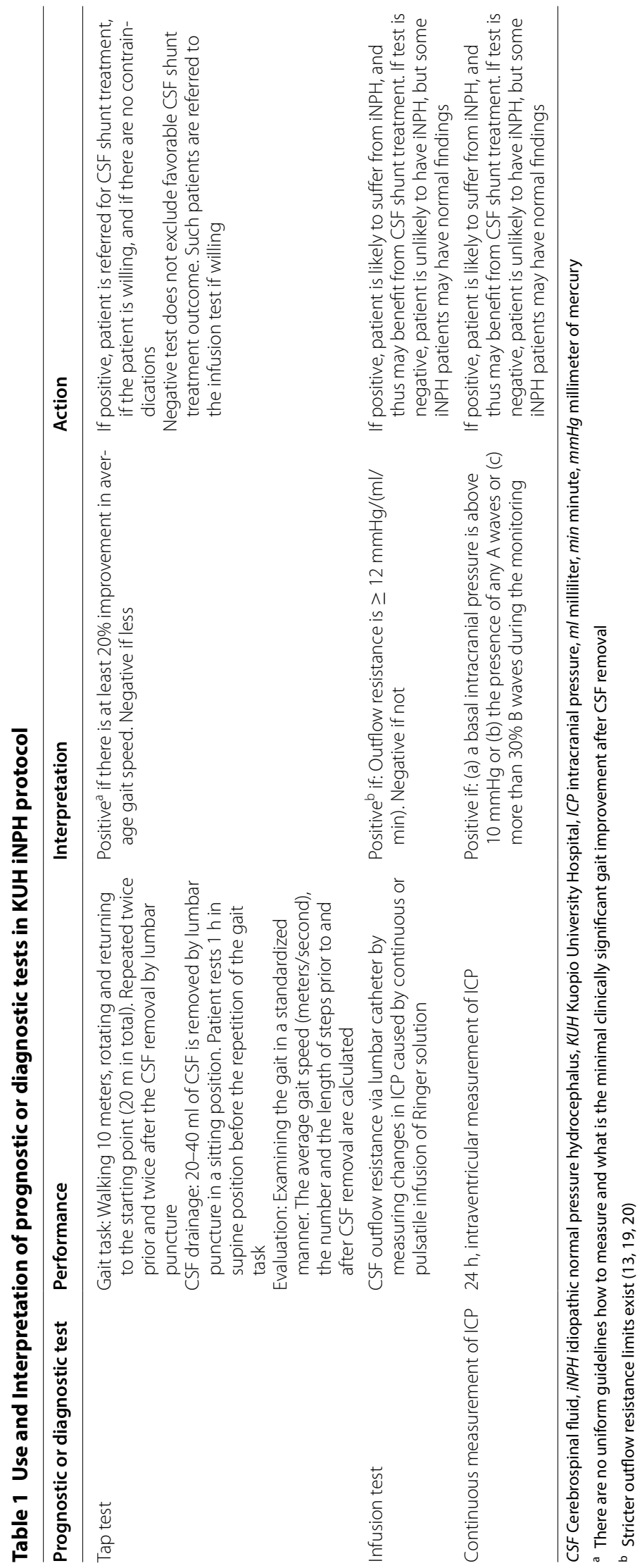


In early 2010, a three-step prognostic test protocol was launched (Fig. 1, Table 1). The use and interpretation of different prognostic or diagnostic tests used in $\mathrm{KUH}$ are presented in Table 1. In the three-step-protocol, an LTT is performed to all patients with suspected iNPH, where at least $20 \%$ improvement in average gait speed in repeated $10-\mathrm{m}$ tests is considered as a positive result (Table 1). In the second phase, those with a negative LTT may undergo lumbar infusion test, where pathological findings (outflow resistance $\geq 12 \mathrm{mmHg} /(\mathrm{ml} / \mathrm{min})$ ) [19] were considered as a positive result. In the third step, participants with a negative finding in both of the abovementioned tests could further undergo a 24-h monitoring of intraventricular pressure (Table 1). Patients with negative findings at any stage of the prognostic testing could still be considered as candidates for shunt surgery based on clinical re-evaluation. Due to the invasive nature of the procedure, the number of patients referred to direct ICP monitoring has decreased over time.

\section{Lumbar tap test}

The LTT is used to temporarily emulate the function of a CSF shunt in order to predict the outcome of treatment [2, 11-13] (Table 2). This was done by examining the gait in a standardized manner before and $1 \mathrm{~h}$ after the removal of 20-40 $\mathrm{ml}$ CSF by lumbar puncture [2, 11, 13]. The KUH procedure for performing the LTT is described in detail in Table 1.

\section{Infusion test}

The infusion test was performed by a neurosurgeon using the Likvor CELDA ${ }^{\circledR}$ System (19). Increased outflow resistance $(\geq 12 \mathrm{mmHg} /(\mathrm{ml} / \mathrm{min})$ [19] was considered to support the diagnosis of probable iNPH.

\section{Shunt surgery}

A ventriculoperitoneal shunt system was used in all patients. The ventricular catheter was placed from either a parieto-occipital or a frontal puncture with the latter being the only applied technique in recent years. The peritoneal catheter was placed via midline- or para-umbilical mini-laparotomy. At the beginning of the study period valves with a fixed pressure setting were used and later the policy was changed to installing adjustable valves in all patients.

\section{Biopsy procedure and immunohistochemistry}

At surgery, three cylindrical cortical brain biopsies of $2-5 \mathrm{~mm}$ in diameter and $3-7 \mathrm{~mm}$ in length, were acquired preceding the insertion of CSF shunt proximal catheter, using biopsy forceps (until 2010) or disposable Temno Evolution $^{\mathrm{R}}$ TT146 biopsy needle (Merit Medical Systems Inc., South Jordan, UT, USA) (since 2010). The insertion point for the catheter was approximately $3 \mathrm{~cm}$ from the midline and anterior to the coronal suture. From all samples, a neuropathologist graded the presence of the immunoreactivity for amyloid-beta $(A \beta)$ and hyperphosphorylated tau ( $\mathrm{HP \tau}$ ) using light microscopy [23]. Patients were then further divided by the presence of pathology of any kind, the A $\beta$ or HPт observed in the frontal cortical biopsy (Table 2).

\section{Evaluation of outcome (3 months postoperatively) Clinical outcome}

A clinically-verified shunt response was assessed by a neurosurgeon at the outpatient clinic. [24] The patient was classified to be responsive to the CSF shunt if any improvement in the core symptoms (gait, cognition and urinary incontinence) was detected [24].

\section{Walking speed improvement}

A positive outcome in walking speed is considered as an improvement of at least $20 \%$. The detailed performance and evaluation of the gait task is described in Table 2.

\section{iNPH Grading Scale}

To assess the severity of the symptoms of iNPH, a modified Finnish version of the 12-point iNPH Grading Scale (iNPHGS) was used [25]. INPHGS is a clinician-rated scale to separately estimate the severity of each of the triad symptoms with a scoring based on interviews with the patients or their caregivers and observations by the physician [25]. Lower scores represent less severe symptoms [25]. It has been estimated that even a reduction in the iNPHGS by a single point results in a clinically observable improvement in the patient's condition [26].

\section{D instrument}

To assess the self-rated Health-related Quality of Life (HRQoL) outcome, a generic utility measurement, 15D instrument was used [27]. The 15D instrument has been recently described in detail in patients with iNPH [10]. The minimal clinically significant improvement in HRQoL, measured by $15 \mathrm{D}$, was considered to be 0.015 [28].

\section{Cognitive impairment}

Cognition was evaluated by using the Mini-Mental State Examination (MMSE). MMSE ranges from 0 to 30, with lower scores indicating a greater cognitive decline [29].

\section{Statistics}

The data was analyzed using the Statistical Package for Social Sciences (SPSS 22 for Windows, Version 22.0. IBM Corp., Armonk, NY, USA). Due to the non-normal distribution, independent-Samples Kruskal-Wallis test 


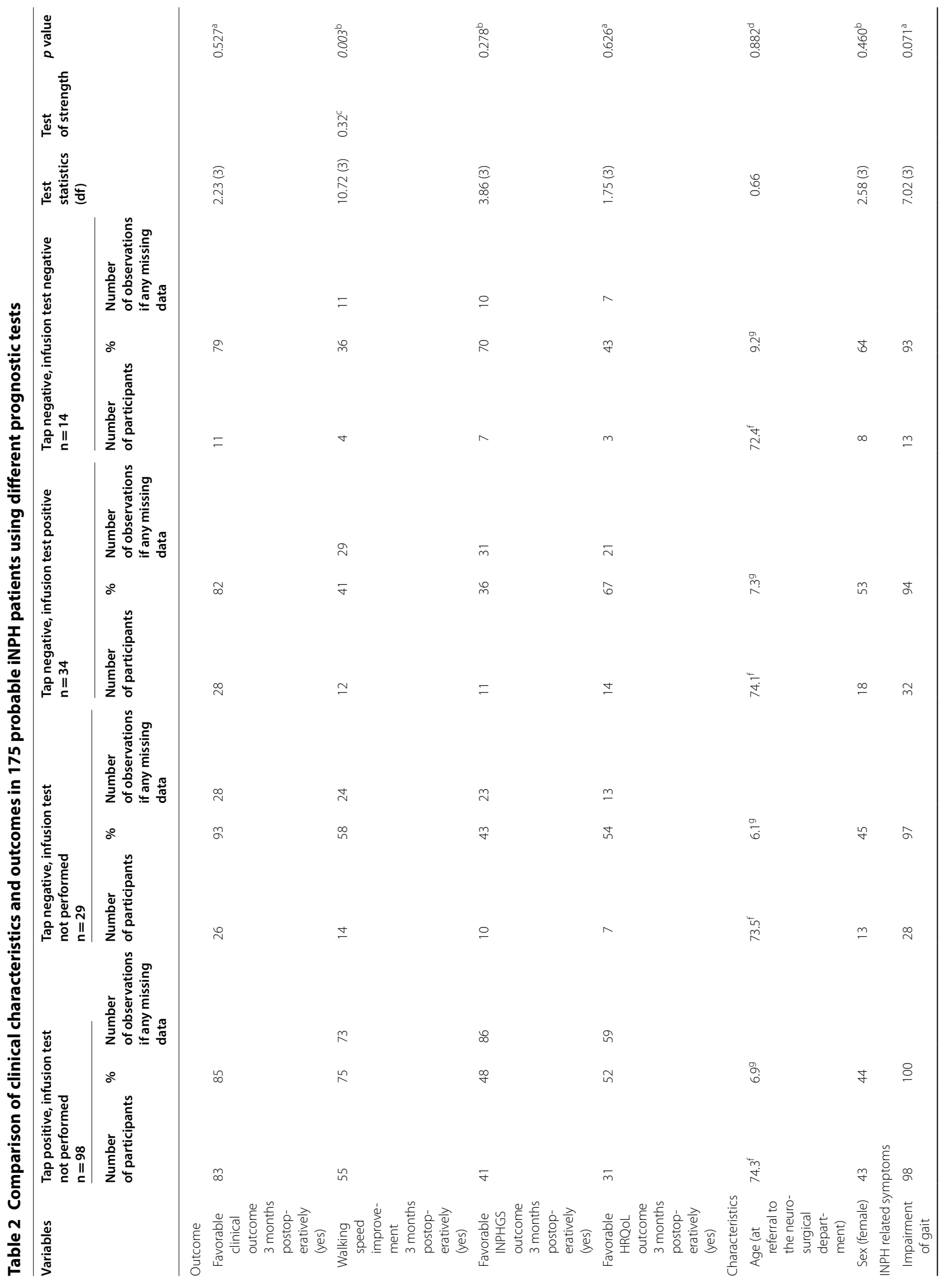




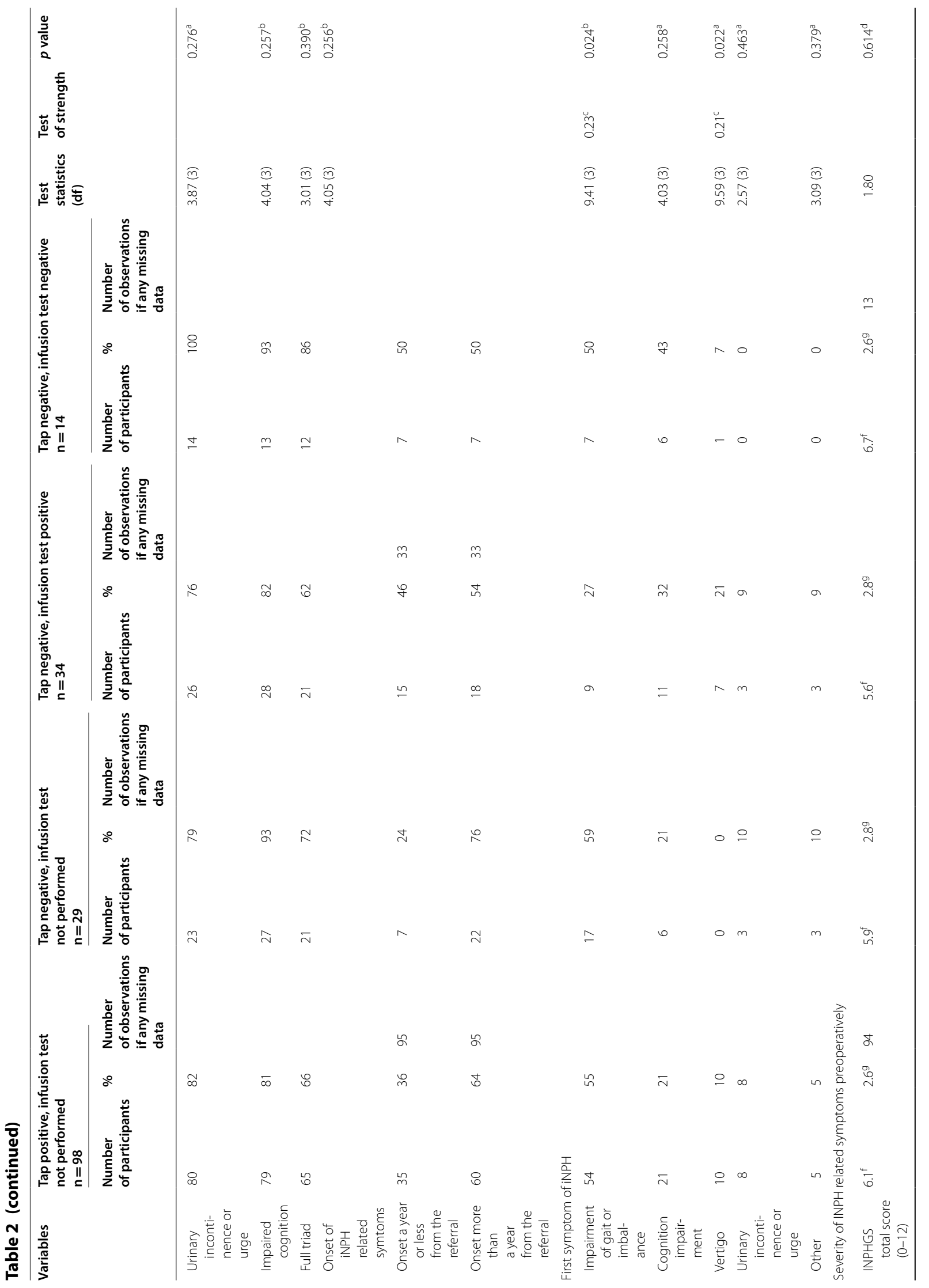


Junkkari et al. Fluids Barriers CNS ～(2019) 16:21

Page 8 of 12

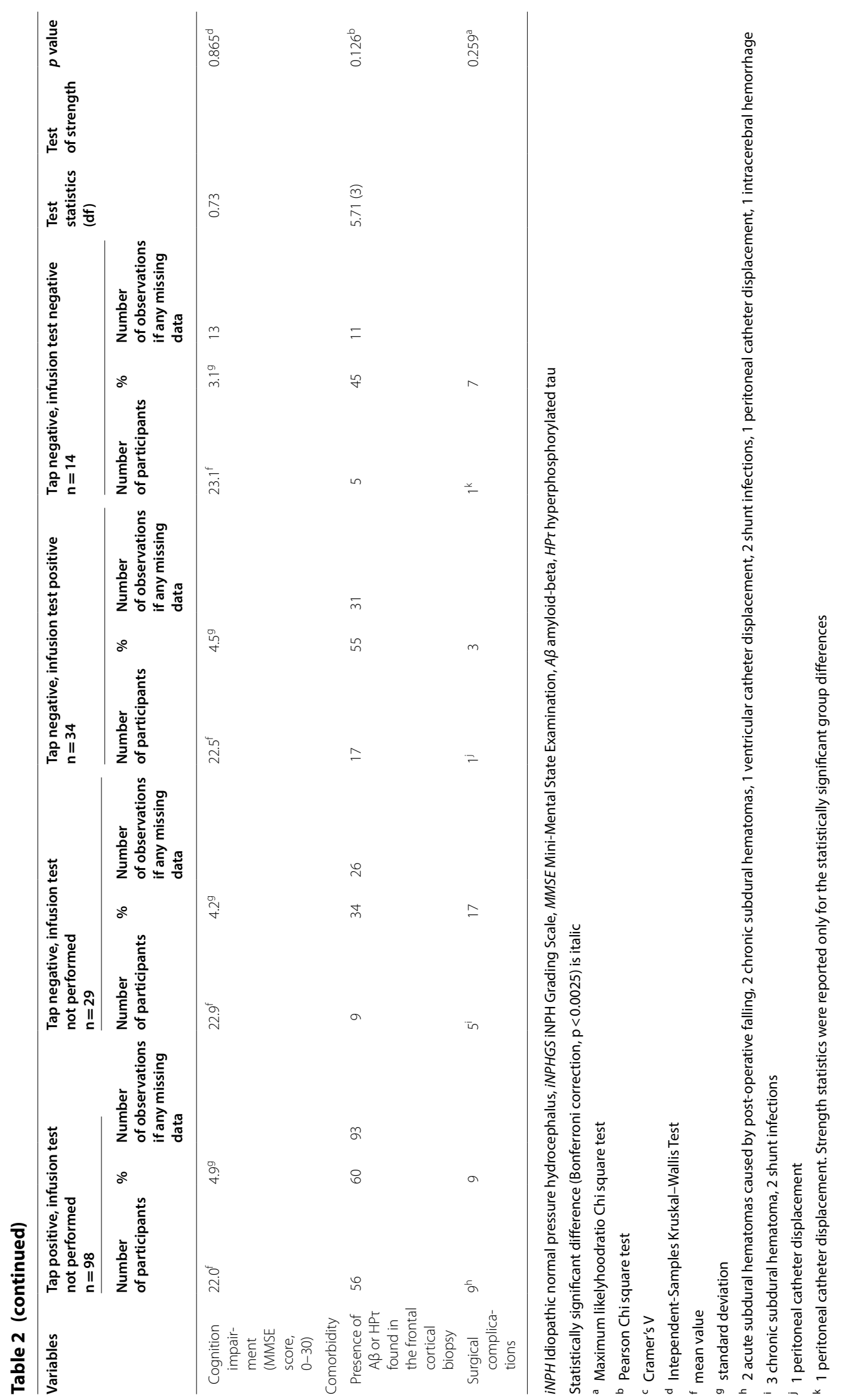


was used in multiple comparisons to estimate group differences in continuous variables. For non-continuous variables Pearson Chi square test was used. If the cell expecteds were 5 or less in more than $20 \%$ of cells, the table was tested with a maximum likelihood ratio Chi square test [30]. Cramer's V was used as a post-test to test the strength of the association between the nominal variables. All tests for significance were two-sided, with probabilities of $<0.05$ accepted as statistically significant. Stricter rejection criterion for $\alpha$ was performed using Bonferroni-correction ( $\mathrm{p}=0.05$ divided by the number of comparisons) to take account multiple comparisons.

\section{Results}

From 341 patients suspected of iNPH, 88 patients were excluded from further research (Fig. 1). The majority of exclusions $(62 / 88)$ were caused by deviation from the protocol's gait assessment guidelines (Table 1). 253 patients with suspected iNPH were included in the study (Fig. 1), 177/253 (70\%) of whom were treated with a CSF shunt (Table 2). Patients were divided into groups according to the prognostic tests used (Fig. 1, Table 2): patients who were shunted on the basis of a positive LTT (98/177, 55\%), negative LTT (29/177, 16\%), negative LTT combined with positive infusion test $(34 / 177,19 \%)$ and to patients who had negative results in both above mentioned tests $(14 / 177,8 \%)$. Only 2 patients $(2 / 177,1.1 \%)$ were referred for ancillary direct invasive ICP monitoring after a negative LTT and infusion test, and thus were not included to statistical analyses.

\section{Outcome of CSF shunting}

The clinical response to CSF shunting was high (7993\%) in all patient groups. A moderate association (Cramer's $\mathrm{V}=0.32$ ) was found between the walking speed improvement rate and the prognostic group $\left(\mathrm{X}^{2}\right.$, $\mathrm{p}=0.003)$ : the walking speed improvement rate was lower if the patient had a negative LTT, and was lowest in patients with both negative LTT and infusion test (4/11, 36\%) (Fig. 1, Table 2). The INPHGS exhibited varying rates of success in each of the four groups that were not significantly different between the prognostic groups. In total, 14 probable or possible iNPH patients were not shunted (Fig. 1). In four cases, severe comorbidities prevented general anesthesia and 10 were due to patient's refusal.

\section{Differences in clinical variables}

The clinical symptomatology of iNPH was rather similar in all four groups (Table 2): gait impairment was present in $93-100 \%$, urinary incontinence or urge was present in $76-100 \%$, and cognitive impairment in $81-93 \%$ of patients. Gait impairment was observed in all patients who had a positive LTT (100\%), whereas urinary incontinence and cognitive impairment were most frequently present in patients with a negative LTT and negative infusion test (100\% and 93\%). Patients who were treated with a CSF shunt regardless of the negative LTT or infusion test tended to present more frequently the full symptom triad (Table 2). These differences were, however, not statistically significant. The patient groups were indifferent in terms of age, cognitive impairment and the severity of iNPH (Table 2). There was no statistically significant difference in the onset of iNPH-related symptoms between the prognostic groups (Table 2).

After Bonferroni-correction for multiple testing, there was no significant difference between the groups when it come to the first presentation of iNPH. Prior to the correction, there was a weak association (Cramer's $V=0.23$ ) between the prognostic group and gait impairment or imbalance as the initial symptom $\left(\mathrm{X}^{2}, \mathrm{p}=0.024\right)$. Prior to the correction, patients who did not undergo an infusion test seemed to have gait impairment or imbalance as the first symptoms more frequently than those to whom the infusion test was performed. Similarly, prior to the Bonferroni-correction, there was a weak association (Cramer's $\mathrm{V}=0.21$ ) between the prognostic group and vertigo as the initial symptom $\left(\mathrm{X}^{2}, \mathrm{p}=0.022\right)$. This atypical presentation seemed more prevalent in patients that underwent infusion testing.

The presence of $A \beta$ or HPt observed in the frontal cortical biopsy varied from 34 to $60 \%$, and no statistically significant differences between the prognostic groups were observed.

Surgical complication rates did not differ between prognostic groups (Table 2). We did not observe significant/permanent complications caused by diagnostic or prognostic tests. Although not systemically collected for this study, we have observed few patients experiencing headache after LTT that required blood patch treatment. Similarly, a small group of patients experienced radiculating pain to lower limb during and shortly after LTT or infusion test, but this pain did not persist and did not require intervention.

Twenty-five percent (62/253) of the study participants had unlikely iNPH, with Alzheimer's disease (AD) as the most frequent diagnosis $(14 / 62,23 \%)$ (Table 3 ). From 62 patients with unlikely iNPH, 11 (11/62, 18\%) had vascular dementia (VAD) or cerebrovascular disease, seven $(7 / 62,11 \%)$ had VAD in addition to $\mathrm{AD}$, six $(6 / 62,10 \%)$ had spinal stenosis, six $(6 / 62,10 \%)$ had cognition impairment or dementia with unspecified etiology (Table 3). All detected conditions are presented in Table 3. 
Table 362 patients with unlikely iNPH referred to KUH iNPH outpatient clinic from 2010 until 2017

\begin{tabular}{lcc}
\hline & Number of patients (\%) & $\begin{array}{c}\text { \% of all } \\
\mathbf{2 5 3} \text { study } \\
\text { participants }\end{array}$ \\
\hline Conditions & & 24.5 \\
Alzheimer's disease & $62(100)$ & 5.5 \\
Vascular dementia or cerebrovascular disease & $14(22.6)$ & 4.3 \\
Alzheimer's disease and vascular dementia & $11(17.7)$ & 2.8 \\
Spinal stenosis & $7(11.3)$ & 2.4 \\
Cognition impairment or dementia with unspecified etiology & $6(9.7)$ & 2.4 \\
Ventriculomegaly (congenital or unclear etiology) & $6(9.7)$ & 1.6 \\
Drug induced Parkinsonism or psychiatric condition & $4(6.5)$ & 1.6 \\
Parkinson's disease (1 idiopathic, 2 vascular) & $4(6.5)$ & 1.2 \\
Lewy's bodies disease & $3(4.8)$ & 0.8 \\
Traumatic brain injury & $2(3.2)$ & 0.8 \\
Frontotemporal dementia & $2(3.2)$ & 0.4 \\
Vertigo & $1(1.6)$ & 0.4 \\
Arthrosis & $1(1.6)$ & 0.4 \\
\hline
\end{tabular}

iNPH Idiopathic normal pressure hydrocephalus, KUH Kuopio University Hospital

\section{Discussion}

The Kuopio iNPH protocol is based on the two existing diagnostic guidelines in terms of the pre-treatment probability, classification and radiological analysis [1, 2], but there are some key differences in the prognostic tests used. While the protocol has emphasis on differential diagnostics, an ELD would have strengthened the prognostic value of the protocol. It is reasonable to assume, that some of the patients with negative LTT in this cohort might have benefitted from drainage test. There is a perplexing question: whether to downgrade the clinical probability for iNPH, as we have done, when competing diagnoses become more likely after the initial probability designation (Fig. 1). One could reasonably argue that the final clinical designation should be made before ancillary testing. Current diagnostic guidelines do not provide an answer to this question, but emphasize ruling out any other medical conditions at the start of classification, and to clinically follow those who exhibit negative prognostic/diagnostic tests $[1,2]$. We argue that an option for reclassification, in addition to unified probability criteria, is needed. An additional difference from the established guidelines is that the cortical biopsy taken at surgery is part of the Kuopio iNPH protocol as a diagnostic and prognostic tool. The biopsy gives additional information for clinicians and helps patient and their families potentially to plan ahead if AD-related pathology is detected.

In clinical practice, the decision whether or not to perform shunt surgery is influenced not only by the results of the prognostic tests, but also by the clinical probability, representation and the accurate identification of iNPH.
A variety of conditions share similar symptoms with iNPH $[1,2,6]$ and may be seen at the outpatient clinic even if a preceding neurological evaluation has been performed (Table 3). This a priori patient selection has had an undoubtable effect on our results, since only a fourth of the patients had a condition other than iNPH. While these conditions were expected, it was interesting to see a heavily skewed distribution: in our cohort, other neurological conditions contributed to most of the differential diagnoses $(74 \%, 46 / 62)$, the second largest group being musculoskeletal conditions $(10 \%, 7 / 62)$ (Table 3). One could argue, that in terms of guideline classification, these patients should remain at least possible iNPH $[1,2]$. We emphasize that while the competing condition was the most likely one in these patients, they can be referred for clinical re-evaluation if a suspicion of iNPH re-emerges.

In our experience, when a thorough differential diagnostics is performed, patients identified to have probable iNPH have a considerable possibility to benefit from CSF shunting even when their LTT and infusion test comes out negative [31]. In cases with more atypical presentation of iNPH, such as patients without gait impairment, infusion testing had a significant value as a differential diagnostic test. It is important to acknowledge, that delaying the treatment in patients with probable iNPH, due to e.g. long waiting times for surgery or unnecessarily extended diagnostic workup, can be harmful $[7,8]$. In our cohort, iNPH patients presenting atypical symptomology significant comorbidities or other potential sources for their symptoms, 
underwent ancillary testing, the clear probable iNPH cases were directed to shunting right after the initial negative LTT. Nevertheless, the general outcome rate in our cohort was similar to that reported in the literature [32].

While a combination of the LTT and the infusion test perform well in identifying potential benefiters of shunt surgery, exclusion criteria based on these tests has not been presented [18]. A patient's neurologic comorbidities, especially $\mathrm{AD}$, may affect the gait response to LTT [31], and therefore the results of the LTT should be evaluated with care. Because the interpretation of the LTT may vary [2, 11-13], one could argue for a higher or a lower gait speed improvement threshold than we have used. While we have used a threshold based on our clinical experience, one could justifiably choose otherwise. Similarly, a longer observation time after LTT might be useful [7, 15, 17]. There exists an unsolved issue regarding the definition of a minimal clinically significant change in gait performance after the LTT. While out of scope to be fully addressed in this paper, we performed receiver operating characteristic (ROC) curve, placing the favorable 3-month iNPHGS -outcome as the binary variable [26]. Analysis did not show threshold for gait speed change (raw and percentage change) in LTT for this outcome indicator in this cohort with limited followup (Additional file 1: Figure S1). Further research in this area is needed.

While the gait performance is, by far, the most objective measure for outcome assessment in iNPH, other outcome indicators should accompany it. Performance in activities of daily living and patient reported outcome measures might enhance clinical evaluation in this regard [10,33], but should not be used alone [33]. Unfortunately, we do not have follow-up information regarding the 14 probable or possible iNPH patients that were not shunted (Fig. 1). iNPH patients that are fit for surgery are encouraged to have shunt surgery after clinical re-evaluation, even after initial refusal.

\section{Conclusions}

Our results emphasize the role of systematic diagnostic and prognostic workup especially in cases with an atypical presentation of iNPH e.g. without gait impairment as the leading symptom. Additional diagnostic testing may be required, but that should not delay adequate care. Active surgical treatment is recommended in patients with a high clinical probability of iNPH. Other neurological conditions contribute most of the differential diagnoses.

\section{Limitations and generalizability}

The cut-off points between positive and negative prognostic tests as well as the performance of the tests vary between published studies. A number of limitations are identified in this study. A longer follow-up time would have strengthened the data. The LTT and the infusion test were performed from different lumbar punctures and ELD was not used in our protocol. Furthermore the neurosurgeon who reviewed the patient postoperatively was not, by rule, independent of the surgery.

\section{Additional file}

Additional file 1: Figure S1. ROC analysis for 3-mo favorable iNPHGS outcome using gait speed change in LTT. Figure Legend: Favorable iNPHGS outcome is a reduction in the iNPHGS total score at least by a single point. Abbreviations: ROC, Receiver operating characteristic; AUC, Area under the curve; INPHGS, iNPH grading scale; iNPH, idiopathic normal pressure hydrocephalus.

\section{Abbreviations}

iNPH: idiopathic normal pressure hydrocephalus; CSF: cerebrospinal fluid; sNPH: secondary normal pressure hydrocephalus; NPH: normal pressure hydrocephalus; KUH: Kuopio University Hospital; CT: computed tomography; MRI: magnetic resonance imaging; LTT: lumbar tap test; TUG: timed up and go-test; ICP: intracranial pressure; iNPHGS: iNPH Grading Scale; MMSE: MiniMental State Examination; HPt: hyperphosphorylated tau; A $\beta$ : amyloid-beta; AD: Alzheimer's disease; VAD: vascular dementia; PD: Parkinson's disease; iPD: idiopathic PD; LBD: Lewy's bodies disease; CVD: cerebrovascular disease; ml: milliliter; min: minute; mmHg: millimeter of mercury; ROC: receiver operating characteristic.

\section{Acknowledgements \\ We want to thank Marita Parviainen (Research Nurse) for collection of data and performing data entry.}

\section{Authors' contributions}

AJ, AJL, VEK, ND, HKJ, AMK, TR, ON, MK, TH, JEJ and VL drafted and revised the manuscript for content, contributed to the data analysis and interpreted results. Study was designed by VL and AJ. Study supervision and obtaining study funding $V L$ and JEJ. AJ, AJL, AMK, TR, ND, HKJ, ON, JEJ and VL contributed to the data acquisition. AJ performed the statistical analysis. All authors read and approved the final manuscript.

\section{Funding}

This study was supported by the Kuopio University Hospital; State Research Funding (VTR Fund); and the Finnish Medical Foundation.

\section{Availability of data and materials}

The anonymized datasets used and/or analyzed during the current study are available from the corresponding author upon reasonable request.

\section{Ethics approval and consent to participate}

The permission for the research was received from the Research Ethics Board of the Kuopio University Hospital (KUH). Informed consent was obtained from every patient or a legally authorized representative before undergoing evaluations.

\section{Consent for publication}

Personal information is de-identified in this manuscript.

\section{Competing interests}

The authors declare that they have no competing interests. 


\section{Author details}

${ }^{1}$ Neurosurgery of NeuroCenter, Kuopio University Hospital (KUH) and University of Eastern Finland (UEF), POB 100, 70029 Kuopio, Finland. ${ }^{2}$ Department of Pathology, KUH and UEF, Kuopio, Finland. ${ }^{3}$ Neurology of NeuroCenter, KUH and UEF, Kuopio, Finland. ${ }^{4}$ Unit of Clinical Neuroscience, Neurosurgery, University of Oulu, Oulu, Finland. ${ }^{5}$ MRC Oulu, Oulu University Hospital, Oulu, Finland.

Received: 25 March 2019 Accepted: 24 June 2019

Published online: 25 July 2019

\section{References}

1. Relkin N, Marmarou A, Klinge P, Bergsneider M, Black PM. Diagnosing idiopathic normal-pressure hydrocephalus. Neurosurgery. 2005;57(3 Suppl):S4-16 (Discussion ii-v).

2. Mori E, Ishikawa M, Kato T, Kazui H, Miyake H, Miyajima M, et al. Guidelines for management of idiopathic normal pressure hydrocephalus: second edition. Neurol Med Chir. 2012:52(11):775-809.

3. Espay AJ, Da Prat GA, Dwivedi AK, Rodriguez-Porcel F, Vaughan JE, Rosso $\mathrm{M}$, et al. Deconstructing normal pressure hydrocephalus: ventriculomegaly as early sign of neurodegeneration. Ann Neurol. 2017;82:503-13.

4. Snijders AH, van de Warrenburg BP, Giladi N, Bloem BR. Neurological gait disorders in elderly people: clinical approach and classification. Lancet Neurol. 2007;6(1):63-74

5. Koivisto AM, Kurki MI, Alafuzoff I, Sutela A, Rummukainen J, Savolainen $\mathrm{S}$, et al. High risk of dementia in ventricular enlargement with normal pressure hydrocephalus related symptoms1. J Alzheimers Dis. 2016;52:497-507.

6. Malm J, Graff-Radford NR, Ishikawa M, Kristensen B, Leinonen V, Mori E, et al. Influence of comorbidities in idiopathic normal pressure hydrocephalus - research and clinical care. A report of the ISHCSF task force on comorbidities in INPH. Fluids Barriers CNS. 2013;10(1):22-8118-10-22.

7. Kazui H, Miyajima M, Mori E, Ishikawa M, SINPHONI-2 Investigators. Lumboperitoneal shunt surgery for idiopathic normal pressure hydrocephalus (SINPHONI-2): an open-label randomised trial. Lancet Neurol. 2015;14(6):585-94.

8. Andren K, Wikkelso C, Tisell M, Hellstrom P. Natural course of idiopathic normal pressure hydrocephalus. J Neurol Neurosurg Psychiatry. 2014;85(7):806-10.

9. Kahlon B, Sjunnesson J, Rehncrona S. Long-term outcome in patients with suspected normal pressure hydrocephalus. Neurosurgery. 2007:60(2):327-32 (Discussion 332)

10. Junkkari A, Hayrinen A, Rauramaa T, Sintonen H, Nerg O, Koivisto AM, et al. Health-related quality-of-life outcome in patients with idiopathic normal-pressure hydrocephalus - a 1-year follow-up study. Eur J Neurol. 2017;24(1):58-66.

11. Williams MA, Malm J. Diagnosis and treatment of idiopathic normal pressure hydrocephalus. Continuum (Minneap Minn). 2016;22(2 Dementia):579-99.

12. Wikkelso C, Hellstrom P, Klinge PM, Tans JT, European iNPH Multicentre Study Group. The European iNPH Multicentre Study on the predictive values of resistance to CSF outflow and the CSF Tap Test in patients with idiopathic normal pressure hydrocephalus. J Neurol Neurosurg Psychiatry. 2013;84(5):562-8

13. Marmarou A, Bergsneider M, Klinge P, Relkin N, Black PM. The value of supplemental prognostic tests for the preoperative assessment of idiopathic normal-pressure hydrocephalus. Neurosurgery. 2005;57(3):S1728 (Discussion ii-v).

14. Thakur SK, Serulle Y, Minskin NP, Rusinek H, Golomb J, George AE. Lumbar puncture test in normal pressure hydrocephalus: does the volume of CSF removed affect the response to tap? AJNR Am J Neuroradiol. 2017:38(7):1456-60.
15. Onder $\mathrm{H}$, Hanalioglu S. Does the volume of CSF removed affect the response to a tap in normal pressure hydrocephalus? AJNR Am J Neuroradiol. 2018:39(1):E5-6.

16. Ishikawa M, Yamada S, Yamamoto K. Early and delayed assessments of quantitative gait measures to improve the tap test as a predictor of shunt effectiveness in idiopathic normal pressure hydrocephalus. Fluids Barriers CNS. 2016:13(1):20.

17. Yamada S, Ishikawa M, Miyajima M, Nakajima M, Atsuchi M, Kimura T, et al. Timed up and go test at tap test and shunt surgery in idiopathic normal pressure hydrocephalus. Neurol Clin Pract. 2017;7(2):98-108.

18. Raneri F, Zella M, Di Cristofori A, Zarino B, Pluderi M, Spaqnoli D. Supplementary tests in idiopathic normal pressure hydrocephalus: a singlecenter experience with a combined lumbar infusion test and tap test. World Neurosurg. 2017;100:567-74

19. Malm J, Jacobsson J, Birgander R, Eklund A. Reference values for CSF outflow resistance and intracranial pressure in healthy elderly. Neurology. 2011;76(10):903-9.

20. Eklund A, Smielewski P, Chambers I, Alperin N, Malm J, Czosnyka M, et al. Assessment of cerebrospinal fluid outflow resistance. Med Biol Eng Comput. 2007:45(8):719-35.

21. Eide PK, Sorteberg W. Outcome of surgery for idiopathic normal pressure hydrocephalus: role of preoperative static and pulsatile intracranial pressure. World Neurosurg. 2016;86:186-193.e1.

22. Luikku AJ, Hall A, Nerg O, Koivisto AM, Hiltunen M, Helisalmi S, et al. Multimodal analysis to predict shunt surgery outcome of 284 patients with suspected idiopathic normal pressure hydrocephalus. Acta Neurochir (Wien). 2016:158(12):2311-9.

23. Seppala TT, Nerg O, Koivisto AM, Rummukainen J, Puli L, Zetterberg H, et al. CSF biomarkers for Alzheimer disease correlate with cortical brain biopsy findings. Neurology. 2012;78(20):1568-75.

24. Koivisto AM, Alafuzoff I, Savolainen S, Sutela A, Rummukainen J, Kurki $M$, et al. Poor cognitive outcome in shunt-responsive idiopathic normal pressure hydrocephalus. Neurosurgery. 2013;72(1):1-8 (Discussion 8).

25. Kubo Y, Kazui H, Yoshida T, Kito Y, Kimura N, Tokunaga H, et al. Validation of grading scale for evaluating symptoms of idiopathic normal-pressure hydrocephalus. Dement Geriatr Cogn Disord. 2008;25(1):37-45.

26. Ishikawa M, Yamada S, Yamamoto K. Early and delayed assessments of quantitative gait measures to improve the tap test as a predictor of shunt effectiveness in idiopathic normal pressure hydrocephalus. Fluids Barriers CNS. 2016:13(1):20

27. Sintonen $\mathrm{H}$. The $15 \mathrm{D}$ instrument of health-related quality of life: properties and applications. Ann Med. 2001:33(5):328-36.

28. Alanne S, Roine RP, Rasanen P, Vainiola T, Sintonen H. Estimating the minimum important change in the $15 \mathrm{D}$ scores. Qual Life Res. 2014;24:599-606

29. Folstein MF, Folstein SE, McHugh PR. Mini-mental state. A practical method for grading the cognitive state of patients for the clinician. J Psychiatr Res. 1975;12(3):189-98.

30. McHugh ML. The Chi square test of independence. Biochem Med (Zagreb). 2013;23(2):143-9.

31. Allali G, Assal F. Brain comorbidities in normal pressure hydrocephalus. Eur J Neurol. 2018;25(8):e94

32. Toma AK, Papadopoulos MC, Stapleton S, Kitchen ND, Watkins LD. Systematic review of the outcome of shunt surgery in idiopathic normal pressure hydrocephalus. Acta Neurochir (Wien). 2013;155(10):1977-80.

33. Junkkari A, Roine RP, Luikku A, Rauramaa T, Sintonen $\mathrm{H}$, Nerg O, et al. Why does the health-related quality of life in idiopathic normal-pressure hydrocephalus fail to improve despite the favorable clinical outcome? World Neurosurg. 2017;108:356-66.

\section{Publisher's Note}

Springer Nature remains neutral with regard to jurisdictional claims in published maps and institutional affiliations. 